\title{
Diagnóstico para la atención de la lengua totonaca en la Universidad Veracruzana Intercultural en \\ México
}

Daisy Bernal Lorenzo ${ }^{1}$

\section{Resumen}

Este manuscrito presenta los resultados de un diagnóstico para la atención de la lengua totonaca, que deriva de la investigación Planificación y políticas del lenguaje para el diseño de programas educativos de náhuatl y totonaco: el caso de la Universidad Veracruzana Intercultural. El trabajo se llevó a cabo del año 2015 al 2017. Se encuestó a 64 profesionistas totonacohablantes; a 18 entidades empleadoras que promueven a hablantes de lenguas originarias; y se entrevistó a 16 especialistas en temas de lengua y educación en contextos interculturales. El resultado orientó a la constitución de un grupo de expertos que discutiera la pertinencia de atender el totonaco mediante el Diplomado Escritura de la Lengua Totonaca, como una primera oferta formativa para los propios totonacohablantes.

Palabras claves: Diagnóstico, Lengua totonaca, Diseño curricular, Política del lenguaje, Educación intercultural.

\section{Talaqstuqni ${ }^{2}$}

\footnotetext{
1 Es docente-investigadora de la Universidad Veracruzana Intercultural, sede regional Totonacapan, de la Universidad Veracruzana en México. Es Responsable de la Orientación de Lenguas de la Licenciatura en Gestión Intercultural para el Desarrollo y se desempeña como docente en el área de lenguas de la Licenciatura en Derecho con Enfoque de Pluralismo Jurídico de esta entidad académica. Pertenece al grupo étnico zapoteco de El Nigromante, Veracruz, que se ubica en el municipio de Playa Vicente. Sus líneas temáticas de investigación se enfocan en los estudios sociolingüísticos, en la didáctica de lenguas y en las políticas lingüísticas en contextos de pueblos indígenas.
}

2 Texto traducido del español al totonaco por el Mtro. Faustino Montes Castañeda. Doctorante de Lingüística Indoamericana por el CIESAS-México, Ciudad de México. 
Uuma taskujut maasiyumaa xtalia'qtaayat kintachuwinkan xatotonako, wa nikuma taxtuucha nak xataqalhputsan taskujut Planificación y políticas del lenguaje para el diseño de programas educativos de náhuatl y totonaco: el caso de la Universidad Veracruzana Intercultural. Uuma taskujut ey punchuwa' tsukuniit, la'ata ka'ta 2015 chu 2017. Kaaqalhskinkaniit tutumpuxamataati xalaqaqsqalaalan qalhtawaqanaanin wantiku chuwinanquy uuma tachuwin; chu naa kaqalhskinkaniit qalhkuchaaxan maaqalhtawaqanaanin wantiku liiskujmaaqulh xaliqalhtawaqa tachuwin chu xatamaasiyun. Uuma taskujut liiqantaxtucha aama xatamaasiyun Diplomado Escritura de la Lengua Totonaca, wantiku chuwinanquy uuma tachuwin.

Tachuwin: Taputsan, Tachuwin, Diseño curricular, Política del lenguaje, Educación intercultural.

\section{Abstract}

This paper presents the results of a diagnosis of the Totonac language, which comes from the research project: Planificación y políticas del lenguaje para el diseño de programas educativos de náhuatl y totonaco: el caso de la Universidad Veracruzana Intercultural. This work ocurred from 2015 to 2017. Surveys were applied to 64 Totonac professional native speakers; 18 employers who promote the use of indigenous languages; and 16 specialists in the language and education areas who have studied the intercultural contexts were interviewed. This result guided a specialists' group who discussed the expedience of attending to the Totonac as a first formative offer within a Diplomado Escritura de la Lengua Totonaca for its own native speakers.

Keywords: Diagnosis, Totonac Language, Curriculum Design, Language Policy, Intercultural Education. 


\section{Introducción}

México es un país multilingüe. Se hablan 364 variantes lingüísticas, clasificadas en 68 agrupaciones lingüísticas y en 11 familias lingüísticas (INALI, 2009) con respecto a las lenguas indígenas. De entre los estados con mayor presencia de lenguas indígenas, figura Veracruz, en donde se hablan 15 lenguas (AVELI, 2010). La lengua totonaca es la tercera lengua regional más hablada en esta entidad federativa, después del castellano y el náhuatl, pertenece a la familia lingüística totonaco-tepehua (INALI, 2009). El II Conteo de Población y Vivienda 2010 registró 212,131 hablantes de totonaco, de los cuales 105,572 se encuentran en el Estado de Veracruz y 106,559 en el estado de Puebla (Mapa 1).

Mapa 1. Presencia del totonaco en los estados de Veracruz y Puebla

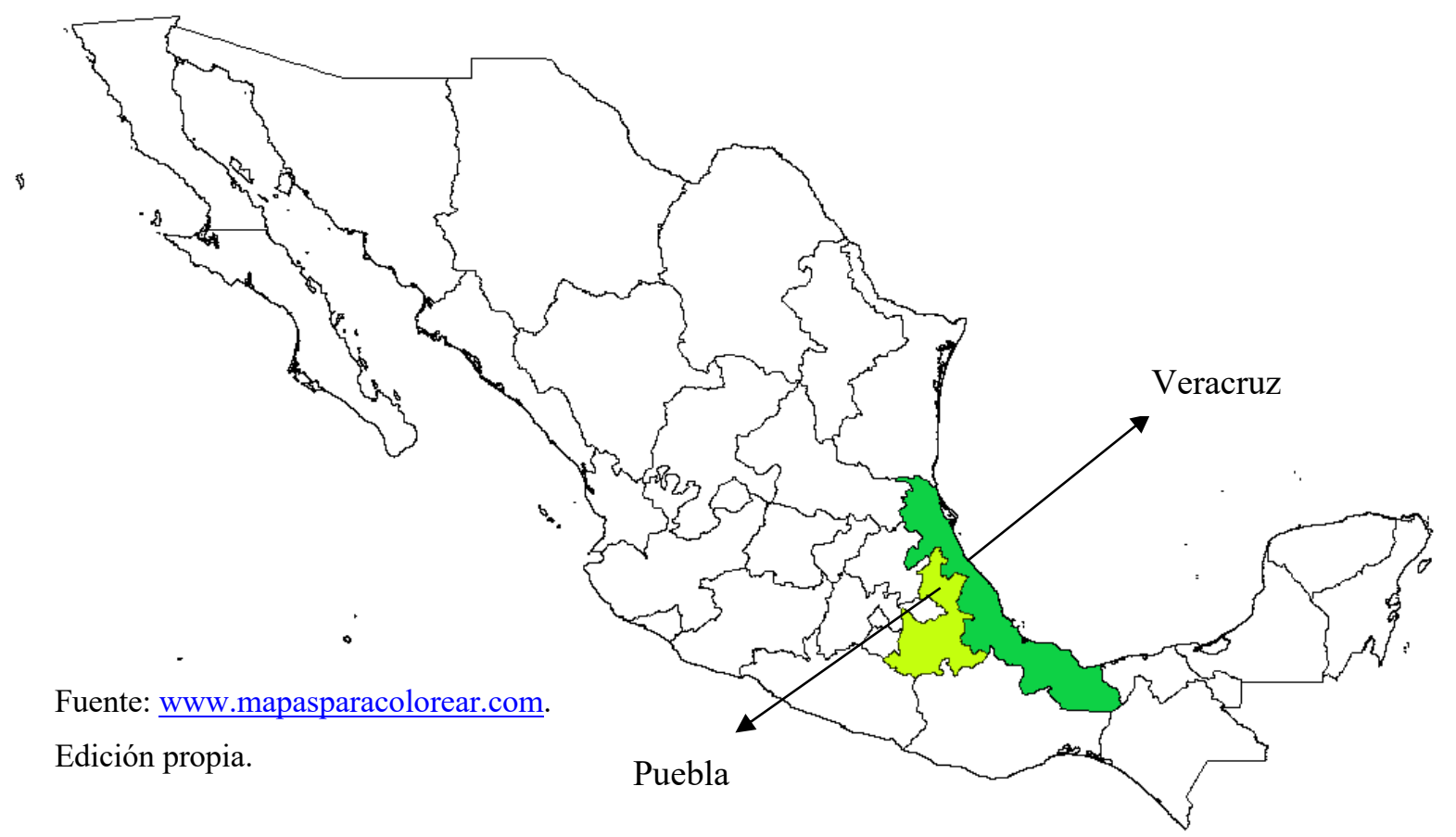

Según García Ramos (2007), existen dos microrregiones que acogen a la mayor parte de la población de hablantes del totonaco. Dichas regiones están comprendidas por quince municipios ubicados entre el Río Tecolutla, el Río Cazones y al noroeste de la sierra de Puebla. La primera región se encuentra en la zona baja de la costa, incluye los municipios de Tihuatlán, Coatzintla, Poza Rica, Papantla, Cazones, Gutiérrez Zamora, Tecolutla y Espinal; y la segunda región se localiza en la zona alta, y se conforma por los municipios de Coyutla, Mecatlán, Filomeno Mata, Coahuitlán, Chumatlán, Coxquihui 
y Zozocolco de Hidalgo, todos del estado de Veracruz.

Mapa 2. Municipios con presencia del totonaco en los estados de Veracruz y Puebla

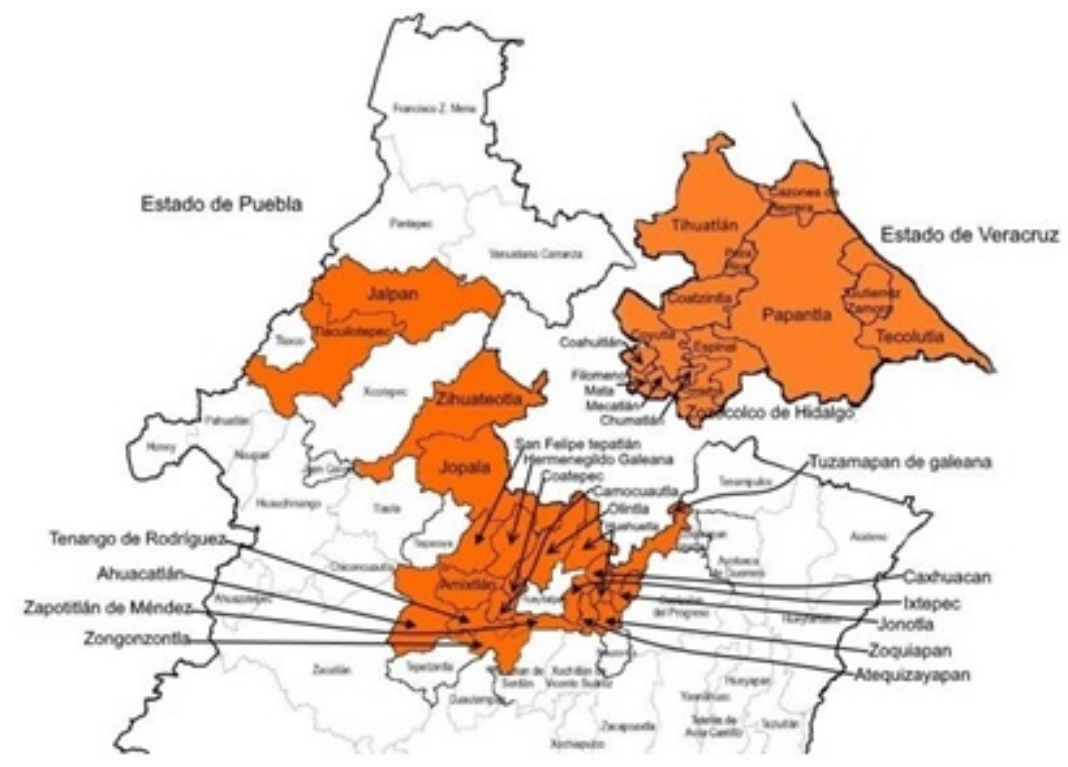

Fuentes: ।

\section{Hernández de la Cruz}

Eusebio

Por su parte, el Instituto Nacional de Lenguas Indígenas (2015) ha registrado que el totonaco se habla en el estado de Puebla, particularmente en los municipios de Ahuacatlán, Amixtlán, Atlequizayán, Camocuautla, Caxhuacan, Coatepec, Cuetzalan del Progreso, Hermenegildo Galeana, Huehuetla, Hueytamalco, Hueytlalpan, Huitzilan de Serdán, Ixtepec, Jonotla, Jopala, Nauzontla, Olintla, San Felipe Tepatlán, Tepango de Rodríguez, Tepetzintla, Tuzamapan de Galeana, Zacapoaxtla, Zapotitlán de Méndez y Zongozotla.

La Universidad Veracruzana Intercultural (UVI), de la Universidad Veracruzana (UV), se ubica en el Golfo de México. Dicha institución ofrece educación superior a jóvenes de zonas indígenas y rurales en la entidad veracruzana, los cuales históricamente han tenido dificultad de acceder a este nivel educativo. Sus sedes regionales se ubican en cuatro municipios estratégicos de la entidad: Ixhuatlán de Madero (Huasteca), Espinal 
(Totonacapan), Tequila (Grandes Montañas) y Mecayapan (Las Selvas).

De la UVI Totonacapan han egresado 71 alumnos licenciados en Gestión Intercultural para el Desarrollo, todos hablantes de totonaco (UVI, 2018). A nivel de impacto institucional, la UVI ha trabajado en la región para que más jóvenes del medio indígena puedan ingresar a sus programas educativos. Sin embargo, aunque algunos de estos jóvenes se consideren totonacos, factores sociales de diversa índole han provocado la interrupción de los procesos de transmisión intergeneracional de la lengua, por lo que muchos de ellos no son hablantes de dicha lengua ${ }^{3}$. La primera generación de estudiantes inició en agosto de 2017 cursando la Licenciatura en Derecho con Enfoque de Pluralismo Jurídico. Hasta el momento, son pocos los alumnos por generación que hablan totonaco, pero se puede observar que los que hablan la lengua se sienten orgullosos de hacerlo, lo cual motiva a que otros expresen que son hablantes o que se consideran totonacos.

La UVI promueve y difunde su diversidad lingüística y cultural desde que sus programas educativos fueron creados en el año de 2005, esto como parte de sus micropolíticas lingüísticas. Baldauf (2010) define a la política y planificación del lenguaje como "la planificación - a menudo a gran escala y nacional, la que usualmente emprenden los gobiernos-que tiene como propósito influir en -si no cambiar-las formas de hablar o las prácticas de alfabetización dentro de una sociedad" (p. 82). Asimismo, este autor refiere a la política como las declaraciones de intención, y a la planificación como la instrumentación en respuesta a esa política. Siguiendo este binomio conceptual, planteo aquí que, en términos regionales y locales, podemos hablar de micropolíticas del lenguaje como las declaraciones (a nivel discursivo) de hablantes de una lengua en particular, de forma individual o colectiva, que proyectan iniciativas $y$ acciones que favorecen el mantenimiento y actualización de una lengua.

Actualmente, los planes de estudios de la Licenciatura en Gestión Intercultural para el Desarrollo (LGID) y la Licenciatura en Derecho con Enfoque de Pluralismo Jurídico (LDEPLUJ) ofrecen Experiencias Educativas (EE) de lenguas, aunque la LGID sólo cuenta con una Experiencia Educativa (EE) de Lengua Local, a diferencia de la LDEPLUJ que cuenta con más EE para el desarrollo de habilidades lingüísticas y comunicativas en totonaco, tanto para los que la cursan como segunda lengua (L2), como los hablantes de la misma que no escriben ni leen en totonaco. Hasta ahora, uno de los logros ha sido la

\footnotetext{
${ }^{3}$ La discriminación y/o maltrato que sus padres y/o abuelos recibieron en la escuela, orillaron el abandono de la lengua a nivel familiar.
} 
sensibilización sobre esta lengua, además, quienes la hablan se animan a usarla en sus procesos de investigación. Lo anterior justifica la necesidad de que la institución oferte e imparta más cursos de totonaco, como L2, ya que esto ayudaría a que los estudiantes incrementen su dominio sobre la lengua y mejoren los procesos de comunicación intercultural.

La UVI ha transitado por varias experiencias para ser congruente con sus micropolíticas lingüísticas, mismas que el propio modelo intercultural declara de manera explícita en documentos oficiales y en los discursos institucionales. Si bien falta mucho por hacer, la UVI ha emprendido proyectos de intervención lingüística y ha buscado de manera permanente responder con coherencia a las formas de atención de la diversidad cultural y lingüística (Figura 1). La institución se preocupa por garantizar los derechos lingüísticos de la comunidad estudiantil, a la cual recibe y educa en sus programas educativos. Por ello, se plantea la planificación lingüística por etapas o fases acorde a la situación sociolingüística de las lenguas reconocidas de facto, entre ellas, el totonaco.

Figura 1. Políticas lingüísticas de la UVI

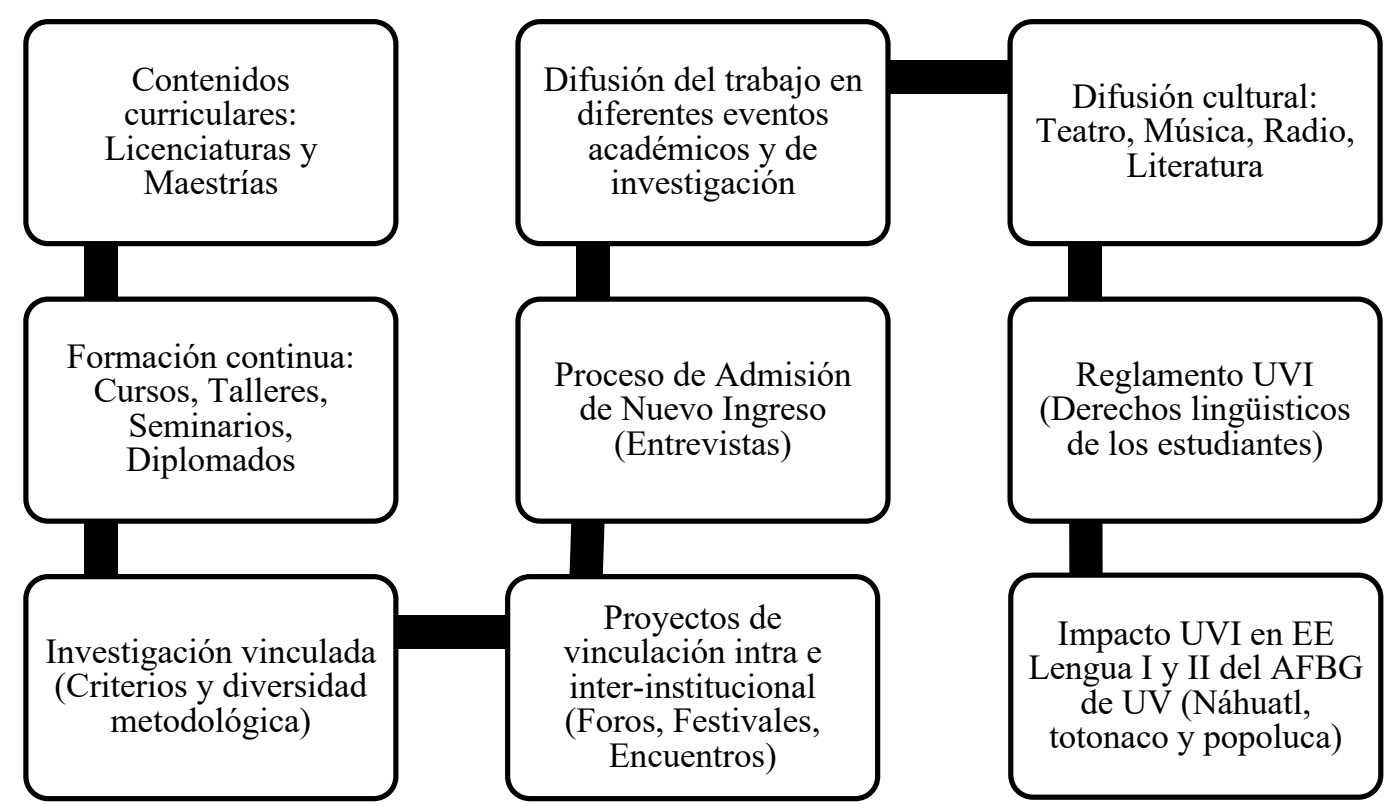

Fuente: Datos propios

Esta planificación académica debe mostrar el impacto que la UVI ha tenido en los 
hablantes y en los demás agentes con los que se vincula, puesto que hacen falta estudios más profundos sobre la situación sociolaboral de profesionistas hablantes de totonaco que indiquen u orienten qué tanto esta lengua les ha servido para encontrar trabajo, qué tipo de formación requieren de cara a la situación laboral en los lugares donde se desempeñan profesionalmente, asimismo, en aquellos profesionistas que requieren de una formación específica o fortalecer sus habilidades básicas a fin de desempeñar mejor su profesión, puesto que el sector educativo es fundamental, así como los de salud, justicia y de la administración pública.

Por ello, este diagnóstico contribuye a la toma de decisiones para encaminar de forma seria los procesos de normalización lingüística del totonaco como objeto de estudio, con la intención de normar ciertos usos académicos de la lengua en el proceso de enseñanza y aprendizaje, mediante procesos formativos sólidos en los ambientes académicos.

\section{Diagnóstico exploratorio}

La metodología y los resultados del presente diagnóstico exploratorio tienen su base en el Informe. Resultado de la Fase Diagnóstica para la Atención del Totonaco 2018. Las técnicas de recogida de información fueron mediante encuestas semiestructuradas y estructuradas, así como entrevistas de preguntas abiertas. Se usó el programa Office Excel, el software SPSS y el Atlas Ti como herramienta de apoyo a la sistematización de la información. Se encuestó a 64 profesionistas hablantes de totonaco (PHT), a 18 empleadores y a 6 especialistas en lengua, educación y/o cultura, en contextos indígenas de México. El trabajo implicó la participación de 17 miembros de la comunidad Uviteca (11 docentes, 1 personal de apoyo, 5 estudiantes de la UVI Totonacapan), 1 docente del Centro de Idiomas UV Poza Rica y 1 egresada de la LGID de la UVI Totonacapan, quienes se integraron en distintos periodos activos del trabajo diagnóstico. Se realizaron reuniones de trabajo de forma presencial y por videoconferencia para discutir y definir el plan de trabajo y avanzar en los acuerdos. Se mantuvo comunicación por correo electrónico, celular, Whatsapp y Facebook, acorde al tipo de participación que cada colaborador decidió contribuir durante el proceso. 
Esquema 1. Proceso metodológico del diagnóstico

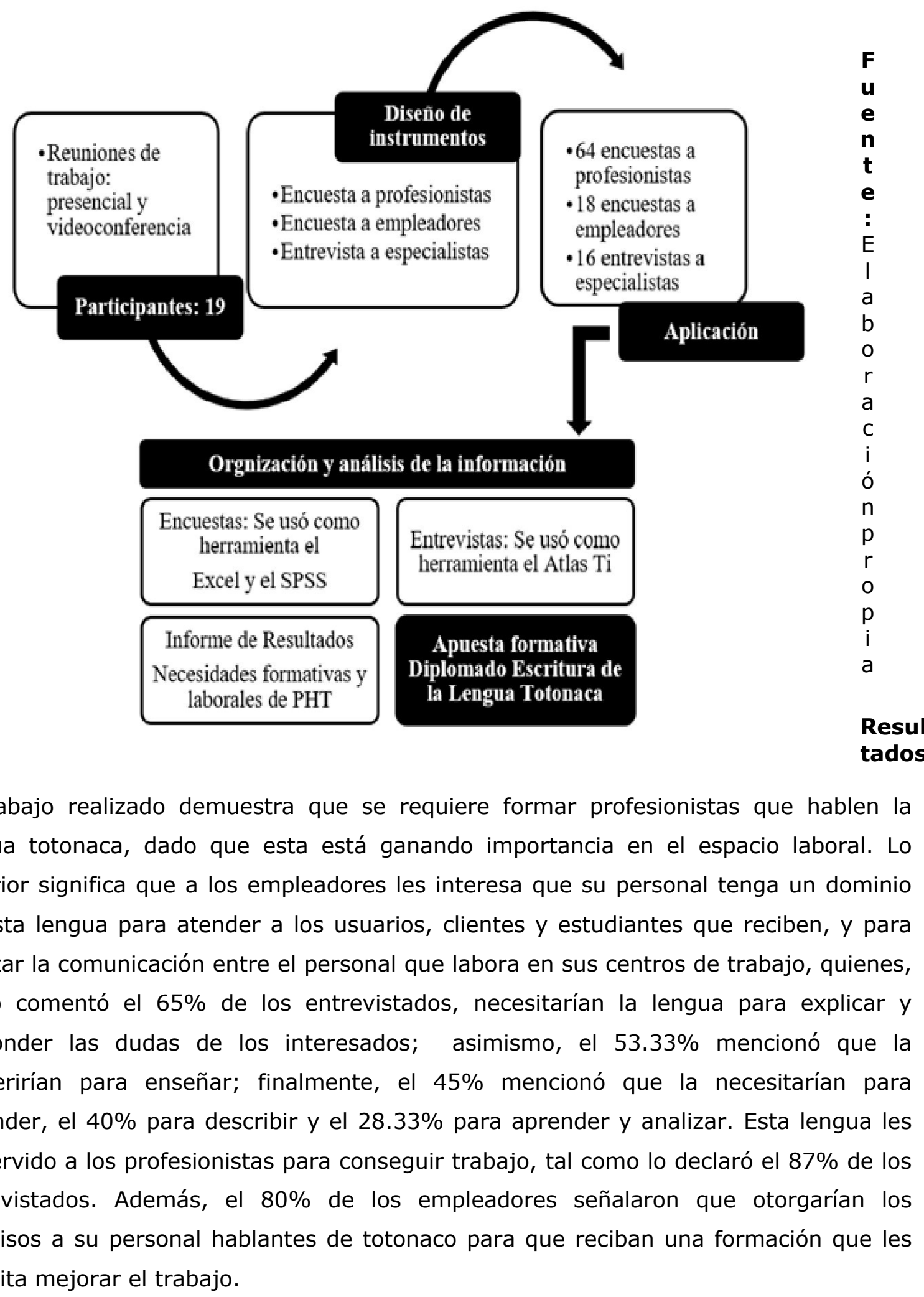

El trabajo realizado demuestra que se requiere formar profesionistas que hablen la lengua totonaca, dado que esta está ganando importancia en el espacio laboral. Lo anterior significa que a los empleadores les interesa que su personal tenga un dominio de esta lengua para atender a los usuarios, clientes y estudiantes que reciben, y para facilitar la comunicación entre el personal que labora en sus centros de trabajo, quienes, como comentó el $65 \%$ de los entrevistados, necesitarían la lengua para explicar y responder las dudas de los interesados; asimismo, el $53.33 \%$ mencionó que la requerirían para enseñar; finalmente, el 45\% mencionó que la necesitarían para entender, el $40 \%$ para describir y el $28.33 \%$ para aprender y analizar. Esta lengua les ha servido a los profesionistas para conseguir trabajo, tal como lo declaró el 87\% de los entrevistados. Además, el $80 \%$ de los empleadores señalaron que otorgarían los permisos a su personal hablantes de totonaco para que reciban una formación que les permita mejorar el trabajo. 
En cuanto a la formación requerida en lo referente a la lengua totonaca, los profesionistas expresaron sus carencias, pues no sólo es importante comunicarse de forma oral y entender para explicar, sino que también es necesario que desarrollen habilidades de escritura y gramática, preservar la lengua, producir textos, generar terminología en salud y conocer la cosmogonía totonaca. En gran parte, lo anterior coincide con lo señalado por los empleadores, puesto que, además de la utilidad que tiene el totonaco en las actividades que realizan los trabajadores, por ejemplo describir, analizar, entender y explicar, es importante que los empleados desarrollen conocimientos necesarios para el desempeño del trabajo, destacando la lingüística, la cultura general, los métodos pedagógicos, los métodos y las técnicas de investigación y la lengua y cultura (Gráfico 1 ).

Gráfico 1: Conocimientos importantes para el desempeño

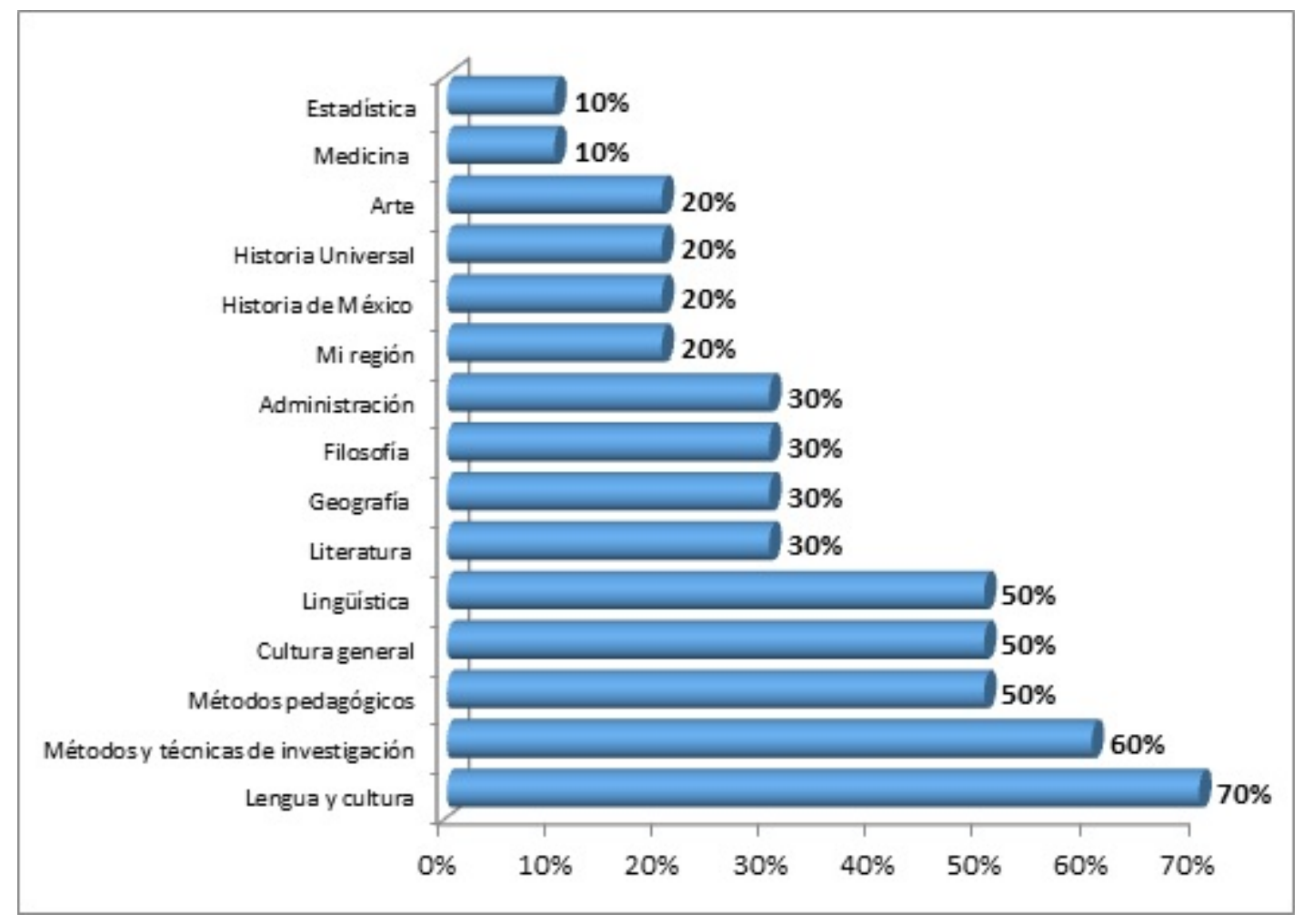

Fuente: UVI, 2018

En lo que respecta al desarrollo de habilidades básicas para que los profesionistas desempeñen mejor su trabajo, destacan el manejo de la computación básica (50\%) y habilidades sociales (60\%), de ahí que la interpretación oral como la lectura y redacción en lengua indígena tengan el mismo porcentaje de importancia (70\%), y sobresaliendo el contar con habilidades de la lengua indígena, como se muestra a continuación: 
Gráfico 2: Habilidades importantes para el desempeño del trabajo

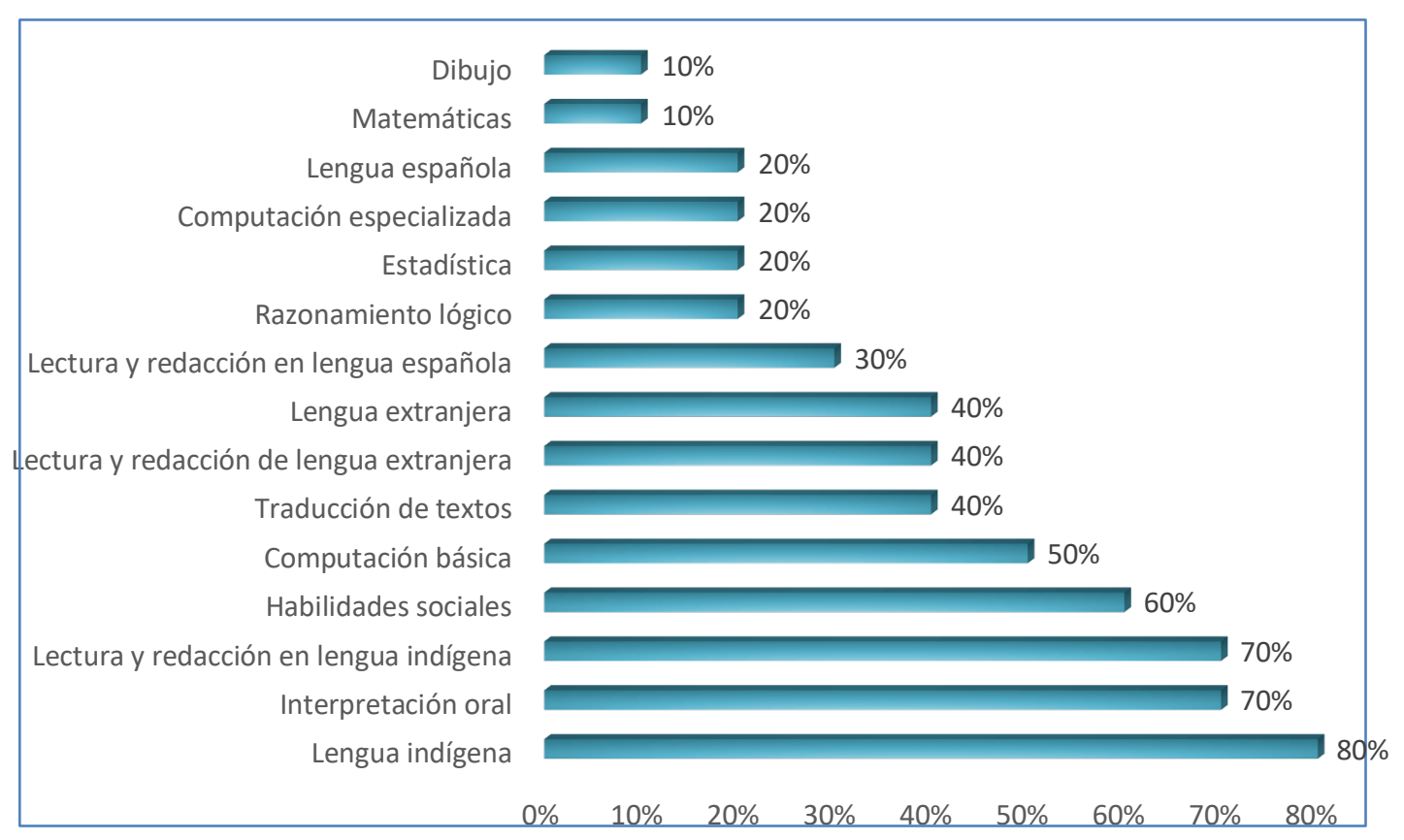

Fuente: UVI (2018)

Las instituciones educativas que realizan diagnósticos pueden prever y planear de mejor manera el tipo de oferta formativa que necesitan los profesionales insertos en un campo laboral, a fin de mejorar su trabajo o aspirar a otros puestos, como se ha venido señalando. Por ello, se les preguntó, tanto a los profesionistas como a los empleadores, qué tipo de oferta requieren. Como se muestra en el siguiente gráfico, un $40 \%$ del primer grupo respondió haciendo mención a una formación en maestría: 
Gráfico 3. Modalidad de mayor interés

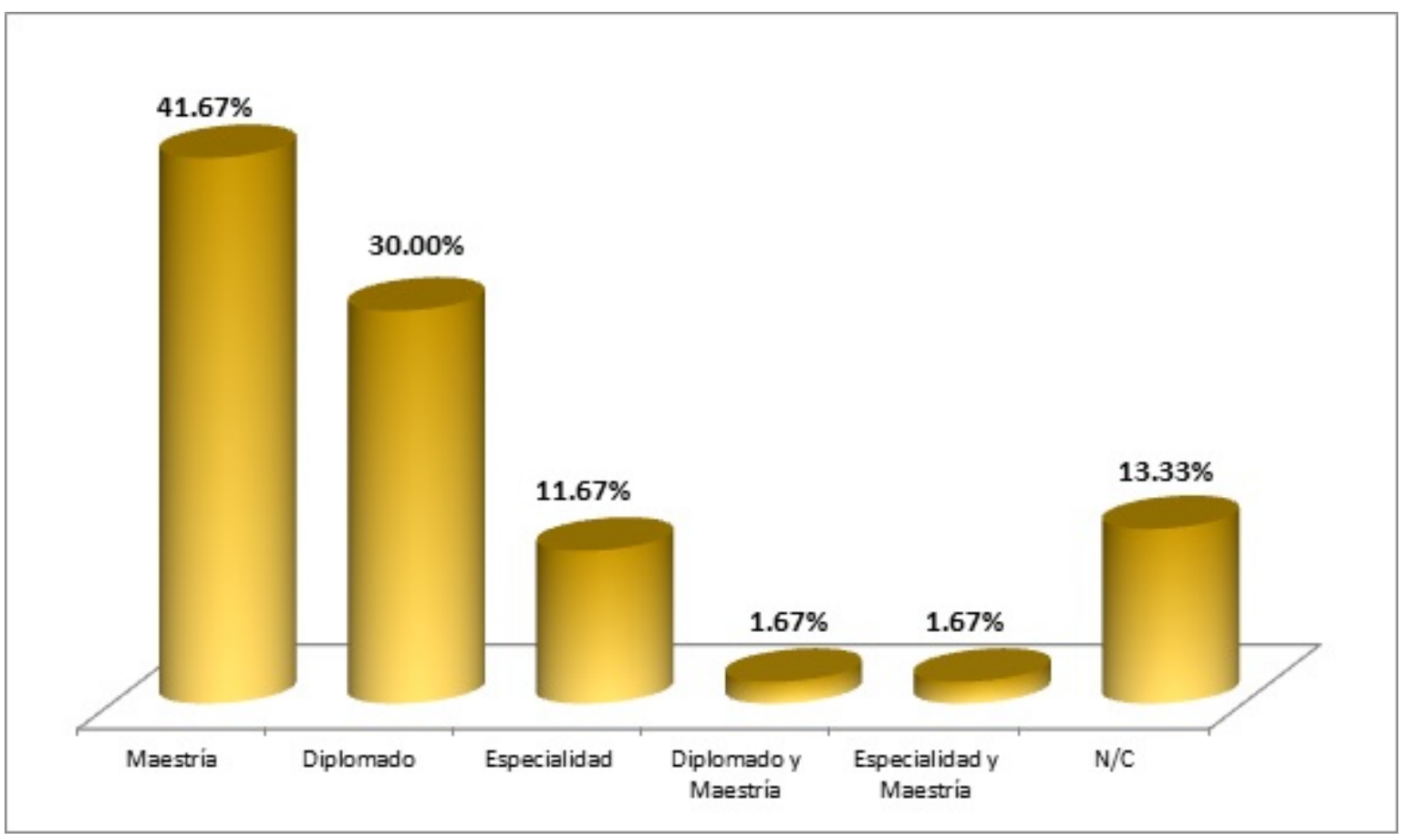

Fuente: UVI, 2018

Los empleadores desean que sus trabajadores tengan un nivel de licenciatura (60\%) y diplomado y doctorado (40\%), como se muestra a continuación:

Gráfico 4. Grado que los empleadores desean que tengan los trabajadores para

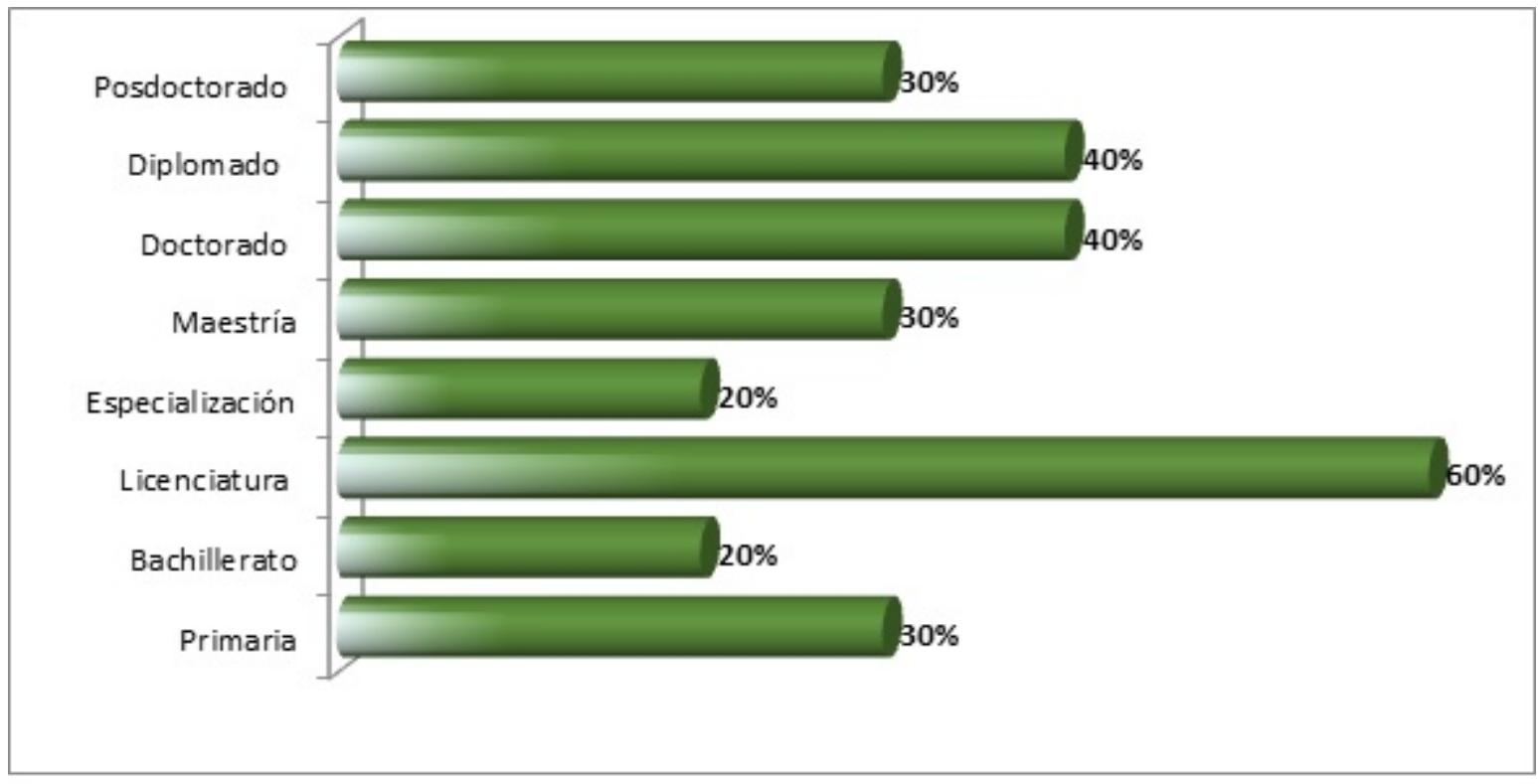


contratarlos

Fuente: UVI (2018)

Los profesionistas manifestaron la utilidad de una formación en la enseñanza y aprendizaje del totonaco $(31.67 \%)$, y su interés formativo se decanta por el tema de lengua y cultura totonaca (23.33\%) para garantizar, mejorar o encontrar un puesto de trabajo. Llama la atención que, como segunda opción formativa, los profesionistas se interesaran en el diplomado como opción y, además, en la misma temática: Enseñanza y aprendizaje del totonaco. Por otra parte, se indagó sobre el sueldo que reciben los profesionistas por el trabajo que desempeñan mensualmente (Gráfico 6), con el propósito de sondear la posibilidad de que puedan cursar un programa formativo, ya que, si perciben un sueldo menor o igual a lo que les costaría la formación educativa deseada, es probable que, si no cuentan con becas o algún tipo de apoyo, sea muy difícil que puedan continuar con sus estudios.

Gráfico 6. Salario mensual percibido por los profesionistas

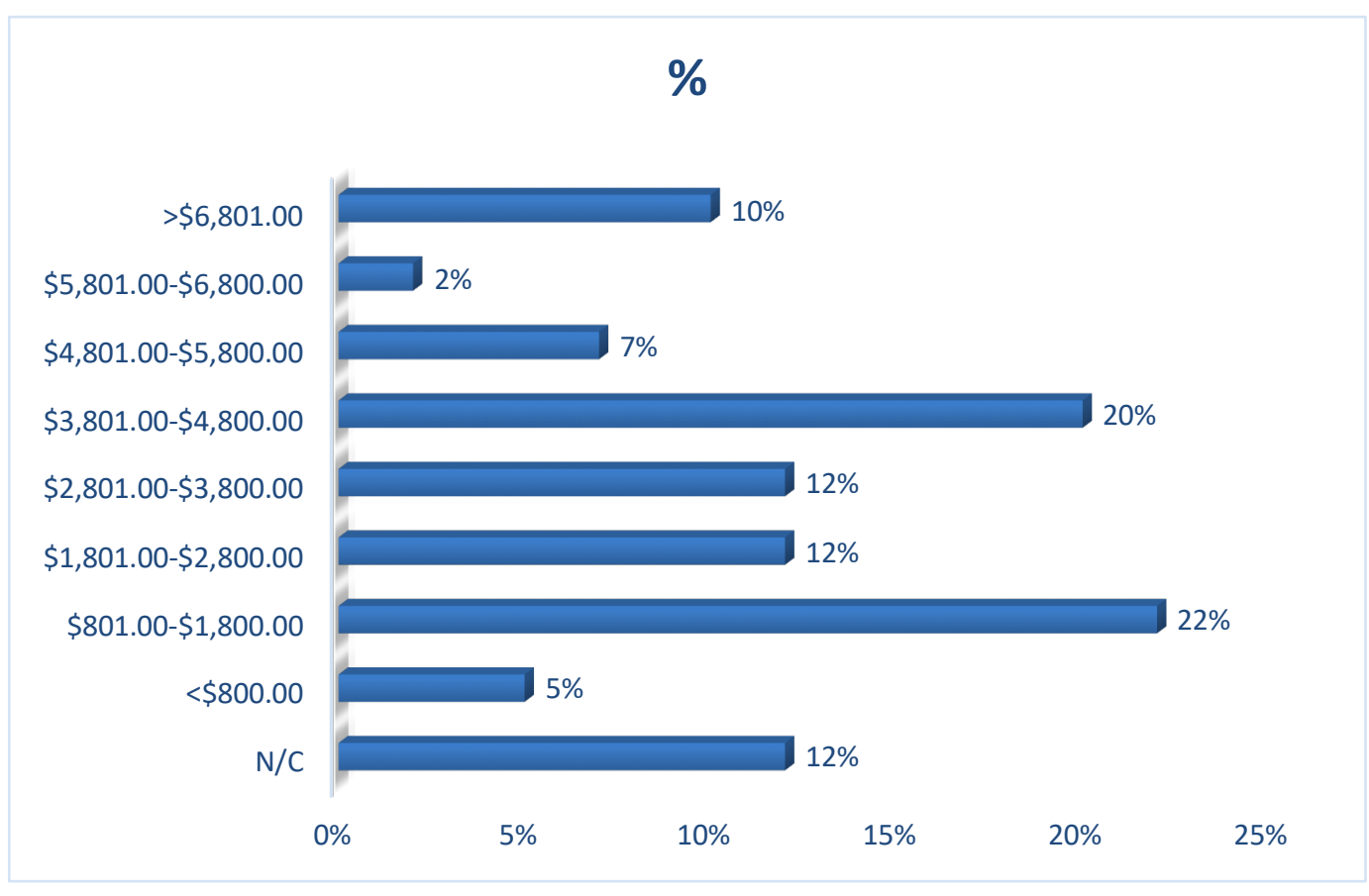

Fuente: UVI (2018) 
Los empleadores ven necesario que sus trabajadores cuenten con un nivel de lectoescritura, lo cual se justifica por el tipo de funciones y tareas que desarrollan en los puestos de trabajo. Por ejemplo, es fundamental que realicen actividades como traducción de materiales de difusión, divulgación, guiones radiofónicos y educativos para la promoción de la salud; también es importante la interpretación, pues requieren de la asistencia de intérpretes-traductores para hacer cumplir la Ley de Derechos y Culturas Indígenas del Estado de Veracruz, así como para generar una cultura de garantía de los derechos lingüísticos en un nivel de educación superior, por ejemplo, la presentación de documentos recepcionales en lenguas indígenas para obtener grados de titulación a nivel universitario.

Por su parte, los especialistas son figuras indispensables para valorar sus opiniones como expertos en el campo de conocimiento en el que se trabaja para una Nueva Oferta Educativa (NOE), así como para hacer viable el trabajo en un diseño de programa formativo para atender la lengua totonaca. Existe una congruencia con lo que compartieron los profesionistas y empleadores. Se aprecia que estas figuras saben que se ha formado a hablantes de lenguas indígenas a nivel nacional, pero sus lenguas no han sido lenguas de instrucción, por lo que la oferta que se proponga puede cursarlo cualquiera que cumpla con los requisitos. Por ejemplo, si fuese una maestría, se pueden beneficiar los egresados de universidades interculturales. Esta posible oferta tendría que enfocarse en temas que coadyuven al desarrollo de habilidades lingüísticas (mayormente en lecto-escritura) y comunicativas, así como para traducir, interpretar y mediar. Si la posibilidad de una maestría fuera inviable, se sugiere transitar hacia este posgrado partiendo del diseño de diplomados que atiendan las necesidades surgidas, pero, en cualquier caso, es primordial trabajar en una base que fortalezca cualquiera de los campos de conocimiento requeridos.

Los especialistas opinaron que las figuras que se formen en una NOE de totonaco pueden ocupar los puestos de trabajo existentes en el país, o bien, crearse otros para elevar la calidad y mejorar el trabajo de manera más humana y profesional en sectores como el educativo, el gubernamental, la administración pública, las empresas privadas, entre otros. Además, los profesionistas pueden desempeñar cargos importantes donde se incentiven y promuevan las lenguas y los derechos lingüísticos, pero ahora con recursos humanos que actúen con pertinencia y que puedan crear proyectos que favorezcan el desarrollo de la lengua y cultura totonaca. 


\section{Conclusiones}

Es importante diseñar una base sólida de formación. Aunque se aspire a posgrados, es trascendental que primero se inicie con cursos, talleres y diplomados que permitan planificar con mayor precisión la atención de la lengua totonaca con pertinencia cultural. Antes de trabajar la traducción y redacción, así como el aprendizaje del totonaco, es fundamental que se inicie con la formación de totonacohablantes para que refuercen y desarrollen las habilidades de la escritura en totonaco, puesto que desde ahí se apreciarán las voluntades y esfuerzos para cultivar una práctica significativa para los procesos de normalización de la lengua totonaca como lenguaje académico universitario, para que esto impacte en los espacios laborales.

Por todo lo anterior, un grupo de estudiosos nativos totonacos, de la coordinación responsable de lenguas de la UVI Totonacapan, así como el Coordinador de Normalización Lingüística, analizaron dichos resultados. Se propuso diseñar el Diplomado en Escritura de la Lengua Totonaca para que la UVI pueda consolidar una postura académica respecto a la escritura de esta lengua (a nivel del sistema gráfico y de las normas ortográficas). Por otra parte, se sugiere que la formación sea de manera permanente para actualizar al profesorado de la UVI que forma a futuros profesionistas en la LGID y en la LDEPLUJ, y mantener una oferta de programas formativos (cursos, talleres, diplomados, etc.) para el público en general que labora en aspectos de la lengua y la cultura totonacas (enseñanza, traducción e interpretación), dado que serán agentes lingüísticos que contribuirán de manera directa a la revitalización y fortalecimiento de la lengua totonaca que aún se habla en la región.

\section{Bibliografía}

Academia Veracruzana de las Lenguas Indígenas (2010). Catálogo de las lenguas indígenas y sus variantes lingüísticas del estado de Veracruz. Xalapa, Veracruz, México: AVELI.

Baldauf, R. B. (2010). "Planificación y política del lenguaje: tendencias recientes, direcciones futuras" en Roland Terborg y Laura García Landa (Coord.): Los retos de la planificación del lenguaje en el siglo XXI, 81-93. México: Universidad Nacional Autónoma de México.

Bernal Lorenzo, D. (2018) (Coord.). Informe Resultado de la Fase Diagnóstica para la Atención del Totonaco 2018. Universidad Veracruzana Intercultural, Espinal, Veracruz. (En revisión).

García Ramos, C. (2007). Diccionario Básico. Totonaco-Español. Español- Totonaco. 
México: Academia Veracruzana de las Lenguas Indígenas y Secretaría de Educación de Veracruz.

Imagui (2012-2018). Estados Mexicanos, recuperado de http://www.mapasparacolorear.com/mexico/mapa-mexico-estadosnombres.png. Consultado el día 8 de enero de 2018.

Instituto Nacional de Lenguas Indígenas (2009). Catálogo de las Lenguas Indígenas Nacionales. Variantes Lingüísticas de México con su autodenominaciones y referencias geoestadísticas, México: INALI.

Instituto Nacional de Lenguas Indígenas (2015). ACERVO DE LENGUAS INDÍGENAS NACIONALES. Recuperado en http://alin.inali.gob.mx/xmlui/handle/123456789/478, consultado el 23 de junio de 2018.

Mapas para Colorear.com. Estados Mexicanos, recuperado en www.mapasparacolorear.com/mexico/mapa-mexico-estados.png. 